\title{
Simplification of the Stratton-Chu Vector Diffraction Integrals and Application to Light Propagation through Curved Surfaces
}

\author{
Shekhar Guha \\ Air Force Research Laboratory, Materials and Manufacturing Directorate, Wright-Patterson Air Force Base, Dayton, Ohio, 45433 \\ Glen D. Gillen \\ Air Force Research Laboratory, Materials and Manufacturing Directorate, Anteon Corporation, Wright-Patterson Air Force Base, Dayton, \\ Ohio, 45433

\begin{abstract}
The Stratton-Chu vector diffraction integrals are expressed in dimensionless forms and then numerically evaluated to describe light propagation originating from a plane and a curved surface without invoking paraxial approximations.

(C) 2005 Optical Society of America

OCIS codes: (050.1940) Diffraction ; (260.1960) Diffraction Theory
\end{abstract}

Given the distribution of the field components of electromagnetic radiation on any surface, the value of the fields at any other point can be obtained in integral forms using the Stratton-Chu theory $[1,2]$ :

$$
\begin{aligned}
& \vec{E}(\vec{r})=\iint\left[i Z_{0} k\left(\hat{n}_{0} \times \vec{H}\right) G\left(\vec{r}, \vec{r}^{\prime}\right)-\left(\hat{n}_{0} \times \vec{E}\right) \times \nabla^{\prime} G\left(\vec{r}, \vec{r}^{\prime}\right)-\left(\hat{n}_{0} \cdot \vec{E}\right) \nabla^{\prime} G\left(\vec{r}, \vec{r}^{\prime}\right)\right] d x^{\prime} d y^{\prime} \\
& \vec{H}(\vec{r})=\iint\left[-i k\left(\hat{n}_{0} \times \vec{E}\right) G\left(\vec{r}, \vec{r}^{\prime}\right) / Z_{0}+\left(\hat{n}_{0} \times \vec{H}\right) \times \nabla^{\prime} G\left(\vec{r}, \vec{r}^{\prime}\right)+\left(\hat{n}_{0} \cdot \vec{H}\right) \nabla^{\prime} G\left(\vec{r}, \vec{r}^{\prime}\right)\right] d x^{\prime} d y^{\prime}
\end{aligned}
$$

where the symbols have their customary meanings [3] and the Green's function $\mathrm{G}$ is defined as

$$
G\left(\vec{r}, \vec{r}^{\prime}\right)=\frac{e^{-i k\left|\vec{r}-\vec{r}^{\prime}\right|}}{4 \pi\left|\vec{r}-\vec{r}^{\prime}\right|} .
$$

The scalar diffraction integrals of Kirchhoff and Rayleigh-Sommerfeld (type I and type II) are included in the first two terms of the Stratton-Chu integrals above. Here, we first express all the electric and magnetic field components in Eqs. (1) and (2) in terms of dimensionless integrals. The integrals are then numerically evaluated using the Kirchhoff approximation for a plane wave incident on a circular aperture. Comparison of the contributions arising from the three terms in Eq. (1) for different distances from the aperture and for different ratios of the aperture radius (a) to the wavelength of light $(\lambda)$ determines the region of validity of the Rayleigh-Sommerfeld and the Kirchhoff diffraction integrals. It is found that although neither of the Rayleigh-Sommerfeld integrals are accurate close to the aperture, the on-axis intensity predicted by the Kirchhoff integral is within $1 \%$ of that calculated using the Hertz vector diffraction theory [4] (which does not invoke the Kirchhoff approximation), even for $a / \lambda=0.5$. For larger values of $a / \lambda$, the deviation between the two are much smaller than $1 \%$.

Next, for a plane wave incident on a cylindrical surface separating two regions of different refractive indices, the double integrals in Eq. (1) were evaluated over the curved surface. Focusing of light close to the geometric focus is observed. It is found that all three integrals in Eq. (1) contribute substantially to the focused intensity. This work extends earlier analysis [5] by including aberration in the focusing system from the start and by not making the assumption of a converging 'spherical' wave. Detailed three dimensional beam distributions in the focal region will be presented.

[1, 2] J. Stratton, Electromagnetic Theory, (McGraw-Hill, New York, 1941), J. A. Stratton and L. J. Chu, "Diffraction theory of electromagnetic waves," Phys. Rev. 56, 99-107, (1939).

[3] Joseph W. Goodman, Introduction to Fourier Optics (Roberts \& Company, Englewood, CO, 2005).

[4] Shekhar Guha and Glen D. Gillen, "Description of light propagation through a circular aperture using nonparaxial vector diffraction theory," Opt. Express 13, 1424-1447 (2005).

[5] A. Boivin and E. Wolf, "Electromagnetic fields in the neighborhood of the focus of a coherent beam," Phys. Rev. 138, 1561-1565 (1965). 